\title{
Interactive comment on "Estimating
} instantaneous sea-ice dynamics from space using the bi-static radar measurements of Earth Explorer 10 candidate Harmony" by \section{Marcel Kleinherenbrink et al.}

Leif Toudal Pedersen (Referee)

Itp@space.dtu.dk

Received and published: 17 February 2021

This is a nice and well written paper describing the potential capabilities of the EE-10 candidate Harmony.

I have a number of minor comments/suggestions: 
L7: speckle tracking -> feature tracking Speckle tracking is a well defined method of tracking mainly applied to relatively slow moving targets such as ice sheets and glaciers. The method is not applicable to sea ice with hours/days between observations, since the backscatter phase will totally decorrelate. Please change this in the entire document.

L15: will -> could

L27: Ricker et al, 2017 is hardly a proper reference for ICESat-2

L37: speckle tracking -> feature tracking

L37: while covering the both poles -> while not covering the poles

L41: Please provide a reference to the claim that instantaneous velocities are an order of magnitude larger than the daily averages in breakup events.

L122-3: A pan-arctic ... - this sentence seems unfinished, please rephrase

L130-: Many terms in the equations are not defined. Please do so.

L138: Doppler -> Doppler shift

L144-45: Please argue how the distances will be kept equal to the accuracy required, in other words that this is a reasonable assumption.

L167: Please argue why it is reasonable to set the volume decorrelation to 1 (for MYice)

L168: difference -> differences

L220-230: These equations are valid for plane parallel layers with no internal scattering

- is that a reasonable assumption?

Printer-friendly version

L252: Please define PSD

Discussion paper

L257: Negative values may me un-physical, but setting them to 0 may lead to a bias if 
the below-zero value came about due to noise? Please discuss.

L279: Please provide a reference for the OceanSAR software package

L294: Please provide a reference to Bartlett's method.

L323: Please rephrase the first line of this sentence - it does not read well.

Figure 3: Some explanation titles in the 3 subplots would be helpful.

Figure 4: A legend inside the plots explaining the full and dotted lines would be good.

L335: speckle tracking -> feature tracking

L342: At the resolution and quality provided here, the subswaths are NOT 'clearly visible' in Fig 5.

L349: flows -> floes

L347-356: This argument is quantitatively somewhat flawed since there are other limiting factors to how much polar coverage S1D will deliver such as SAR duty-cycle and land application requirements. This should be stated/discussed.

\section{L355: L-ROSE -> ROSE-L}

L356: speckle tracking -> feature tracking

L359: speckle tracking -> feature tracking

L364: speckle tracking -> feature tracking

L371-72: This argument seems to assume instant response of the ice drift to wind. This is not what we have in reality. Please argue or modify/explain better what you mean or how this could be achieved.

L384: flows -> floes Figure 8: It might be more illustrative to have the same x-range on the 2 histograms for a better inter-comparison. 
L398: to occur -> to be observed Figure 9: Please explain better why the larger shear are missed by the Harmony estimate

L454-55: Due to ... - this statement needs rewriting.

L458-59: Please clarify that this statement is on your behalf and NOT on behalf of the sea-ice community at large. Other requirements such as more frequent coverage is better obtained using EW.

Some further considerations about the uncertainties in the results presented could be helpful.

Interactive comment on The Cryosphere Discuss., https://doi.org/10.5194/tc-2020-245, 2020. 